\title{
Percepção de ex-moradores de rua sobre sua qualidade de vida
}

\section{Perception of ex-homelles on your quality of life}

\begin{abstract}
RESUMO
Renata Prata Cunha Bernardes Rodrigues'

\section{INTRODUÇÃO}

Os moradores de rua são pessoas que não possuem mais documentos, trabalhos formais nem moradia fixa e também se encontram com vínculos familiares e sociais rompidos.

Objetivo: Avaliação do impacto da saúde bucal na qualidade de vida de ex-moradores de rua que participaram do projeto de extensão de promoção da saúde realizado na Casa de Acolhimento Santa Gemma Galgani, em Uberlândia-MG.

Método: Estudo descritivo, no qual avaliou-se a autopercepção da condição de saúde bucal aferida pelo Oral Health Impact Profile (OHIP-14) e por outras três questões. Após coleta dos dados, atribuiu-se um valor para cada resposta do questionário e realizou-se análise por meio do método aditivo. Participaram 50 indivíduos do gênero masculino acima de 18 anos de idade.

Resultados: $66 \%$ dos indivíduos questionados relataram ter algum problema com seus dentes, $64 \%$ dos indivíduos relataram ter dores em sua boca ou dentes e $56 \%$ se sentem tensos devido a problemas com os dentes, boca ou próteses. Identificou-se que a pior autopercepção da saúde bucal foi associada a condições clínicas bucais ruins, as quais levam a fatores sociais e comportamentais desfavoráveis.

Conclusão: O índice OHIP-14 mostrou a existência de impacto da saúde bucal na qualidade de vida dos ex-moradores de rua, tendo sido maior nas dimensões desconforto psicológico, dor física, incapacidade psicológica e incapacidade social.
\end{abstract}

Thais Diniz Segatto

LÚCIO Borges de ARAÚJol

Palavras-chave: Qualidade de vida; Moradores de rua; Autopercepção de saúde.

'Universidade Federal de Uberlandia (UFU), Uberlandia/MG - Brasil 


\section{INTRODUÇÃO}

Os moradores de rua são pessoas que não possuem mais moradia fixa, documentos, e também se encontram com relações familiares e sociais rompidas. ${ }^{1}$ Possuem menos do que o necessário para atender às necessidades básicas do ser humano, e as questões sociais, políticas, e econômicas são desfavoráveis a esse grupo vulnerável, como exclusão social e pobreza. $^{2}$

O processo de exclusão social gera falta de pertencimento social, falta de perspectivas, dificuldade de acesso à informação e perda da autoestima. Ações Intersetoriais e integradas de promoção, prevenção e reabilitação da saúde são fundamentais para minimizar os problemas e suas consequências no morador de rua, visando sua inclusão social e resgate de cidadania do indivíduo. ${ }^{3}$

É fundamental o papel da comunidade universitária na orientação e participação da educação em saúde da comunidade, em especial, de grupos excluídos. ${ }^{4}$ A interação entre universidade e comunidade é realizada principalmente durante o processo ensinopesquisa-extensão. Nesse processo, o graduando se beneficia por meio de uma formação como cidadão consciente e atuante, comprometido com a saúde e a qualidade de vida das pessoas e a comunidade é favorecida, pois a universidade propõe soluções para os problemas prioritários da população. 5,6 Além disso, a extensão contribui para a formação de profissionais com sólida formação humanística, postura ética, responsabilidade social, visão crítica e atualizada do mundo. ${ }^{7}$

Devido ao importante papel da qualidade de vida como medida de impacto de saúde, pesquisadores têm procurado criar e validar instrumentos que avaliam o impacto das condições de saúde bucal na qualidade de vida dos indivíduos. ${ }^{8}$ O Perfil de Impacto da Saúde Bucal, ou Oral Health Impact Profile (OHIP), está entre os indicadores mais destacados por seu amplo uso. ${ }^{9}$ Quando se associa qualidade de vida a aspectos gerais de saúde e saúde bucal, percebe-se o quanto é fundamental a percepção subjetiva das pessoas relacionada à autoimagem, à apreensão de necessidades e à busca por cuidados odontológicos. ${ }^{10,11}$

A medida da qualidade de vida na população brasileira geraria informações que poderiam ser usadas para o rastreamento e a identificação das necessidades de saúde da população, decisão sobre as prioridades em assistir determinados setores e alocação de recursos, o que contribui para um melhor planejamento em saúde. ${ }^{12}$ Há situações em que ocorre dissonância entre a necessidade de tratamento expressa pelo cirurgiãodentista e aquela referida pelo paciente, o que demonstra existir uma subjetividade em relação às necessidades. ${ }^{13}$

Problemas bucais, como cárie dentária, doença periodontal e perdas dentárias são aspectos clínicos que apresentam impacto negativo na vida cotidiana das pessoas, gerando dificuldades funcionais, desordens na mastigação, na fala e problemas para a sociabilidade, como a insatisfação com a aparência, dificuldade de aceitação social e restrições no acesso ao mercado de trabalho. ${ }^{14,15}$

Com base na literatura, esta pesquisa buscou avaliar, por meio do OHIP-14, o impacto da saúde bucal na qualidade de vida de ex-moradores de rua que participaram do projeto de extensão de promoção da saúde realizado na Casa de Acolhimento Santa Gemma Galgani.

\section{Material e métodos}

O projeto foi realizado na Casa de Acolhimento Fraterno Santa Gemma Galgani, Uberlândia-MG. A casa acolhe no máximo 14 pessoas, devendo ser maiores 
de 18 anos de idade e do sexo masculino. Os moradores de rua são recolhidos pela pastoral da moradia e acolhidos pela casa quando há vagas. Importante salientar que há uma alta rotatividade de moradores, pois a permanência dos mesmos está ligada às regras de boa convivência, sendo proibido o uso de bebidas alcoólicas e de drogas, assim, muitos não conseguem ficar livres do vício e voltam para as ruas. Os moradores de rua acolhidos pela Casa foram designados de exmoradores de rua, pois a partir do momento que são acolhidos, passam a ter um abrigo, alimentação e vestuário, saindo da condição de desmazelo encontrada nas ruas.

Esta pesquisa foi aprovada pelo Comitê de Ética em Pesquisa com Seres Humanos da Universidade Federal de Uberlândia, com o protocolo $n^{\circ} .452 .932$ e executada segundo as normas da resolução 466/2012 do Conselho Nacional de Saúde.

A pesquisa foi desenvolvida dentro de um projeto de extensão e desenvolvido de forma transversal por meio de ações educativopreventivas e da coleta de dados para a avaliação da autopercepção da saúde bucal e do impacto da saúde bucal na qualidade de vida, pelo instrumento OHIP-14, no período de novembro de 2013 a janeiro de 2014.

Durante as ações educativo-preventivas, realizadas pelos alunos extensionistas, temas diversos relacionados à saúde geral, bucal e mental foram abordados e, após essas atividades, a aluna responsável pela pesquisa aplicou o questionário, em local reservado.

Os critérios de inclusão para o estudo foram: ter no mínimo 18 anos de idade e ser residente da Casa de Acolhimento, independentemente do tempo de permanência no local. O instrumento Oral Health Impact Profile, OHIP-14, ${ }^{16}$ foi desenvolvido na Austrália $^{9}$ e empregado em vários países. Este é um dos questionários de autoavaliação de saúde bucal mais usados em função do número reduzido de questões e da facilidade de uso. ${ }^{17}$

$\mathrm{O}$ indicador OHIP considera as consequências sociais dos problemas bucais de acordo com a percepção dos próprios indivíduos afetados. Em sua versão original, é composto por 49 questões. ${ }^{9} \mathrm{Na}$ presente pesquisa utilizou-se uma versão abreviada, o OHIP-14, contendo 14 questões. ${ }^{16} \mathrm{O}$ instrumento contempla sete dimensões do impacto a ser medido: limitação funcional, dor física, desconforto psicológico, incapacidade física, incapacidade psicológica, incapacidade social e deficiência. ${ }^{9}$

O questionário do presente estudo foi composto por 17 perguntas, sendo que as três primeiras foram referentes à autoavaliação da saúde bucal e as 14 perguntas restantes referentes ao OHIP-14. ${ }^{16}$

As possiveis respostas ao OHIP-14 foram em escala Likert com cinco opções: muito frequente, pouco frequente, ocasionalmente, quase nunca ou nunca. Os resultados obtidos foram analisados aplicando-se o método aditivo, por apresentar alto poder discriminatório. ${ }^{18}$ Consiste na soma simples dos valores atribuídos às respostas dadas às questões que compõem o instrumento ( 4 = muito frequente, 3 = pouco frequente, 2 = ocasionalmente, 1 = quase nunca e 0 = nunca), sendo que a escala poderia apresentar uma variação de 0 a 56, possibilitando o cálculo da média. O OHIP-14 foi classificado em dois grupos: sem impacto (ocasionalmente, quase nunca e nunca) e com impacto (pouco frequente e muito frequente), em pelo menos um dos quatorze itens. Quanto maior o valor, tanto maior impacto da saúde bucal na qualidade de vida. ${ }^{19}$ Cada dimensão contém duas questões, sendo limitação funcional (1-2), dor física (3-4), desconforto psicológico (5-6), incapacidade física (7-8), incapacidade psicológica (9-10), incapacidade social (11-12), deficiência (13-14).

A confiabilidade do questionário foi avaliada pelo coeficiente alpha-Cronbach, um dos 
indicadores mais utilizados para verificar a fidedignidade ou consistência interna de um instrumento.

\section{Resultados}

A amostra foi composta por 50 ex-moradores de rua, que durante 0 desenvolvimento da pesquisa estavam na
Casa, independentemente do tempo de permanência na mesma. Os dados coletados foram agrupados em tabelas contendo a frequência e os percentuais das respostas referentes ao questionário de autoavaliação da saúde bucal (Tabelas 1, 2 e 3) e do questionário OHIP-14 (Tabela 4). Segundo as respostas, $46 \%$ classificaram sua saúde como péssima ou ruim e $28 \%$, como excelente ou boa (Tabela 1 ).

Tabela 1 - Percentual das respostas da pergunta A "Como você avalia sua saúde bucal?"

\begin{tabular}{lcc}
\hline & Frequência & Porcentagem \\
\hline Péssima & 7 & 14,0 \\
Ruim & 16 & 32,0 \\
Regular & 13 & 26,0 \\
Boa & 10 & 20,0 \\
Excelente & 4 & 8,0 \\
\hline Total & 50 & 100,0 \\
\hline
\end{tabular}

Em relação a apresentar problemas com os dentes, $66 \%$ dos ex-moradores de rua apresentavam e 34\% não apresentavam (Tabela 2).

Tabela 2 - Percentual das respostas da pergunta B "Você tem algum problema com seus dentes?"

\begin{tabular}{lll}
\hline & Frequência & Porcentagem \\
\hline Não & 17 & 34,0 \\
Sim & 33 & 66,0 \\
Total & 50 & 100,0 \\
\hline
\end{tabular}


Em relação a apresentar problemas com as gengivas, 64\% dos ex-moradores de rua não apresentavam e $36 \%$ apresentavam (Tabela 3 ).

Tabela 3 - Percentual das respostas da pergunta C: "Você tem algum problema com suas gengivas?"

\begin{tabular}{lll}
\hline & Frequência & Porcentagem \\
\hline Não & 32 & 64,0 \\
Sim & 18 & 36,0 \\
Total & 50 & 100,0 \\
\hline
\end{tabular}

Inicialmente, aplicou-se o Teste de Bartllet e o KMO para verificar se a Análise Fatorial (AF) pode ser aplicada no conjunto de dados. O teste de Bartllet apresentou uma estatística qui-quadrado 445,03 e $p<0,0001$, indica que a matriz de correção é estaticamente diferente da matriz identidade e o KMO foi igual 0.752 mostra que o tamanho amostral utilizado é adequado para a análise dos dados. $O$ alpha de Cronbach foi de 0.87 , que indica que há uma boa coerência interna entre os itens avaliados.
Após a análise, foram obtidos quatro fatores, usando-se o critério de se considerar apenas aqueles com autovalores maiores que 1,0 (Critério de Kaiser), como nota-se na Tabela 4. Esses quatro fatores explicam juntos $65,71 \%$ da variação total que são superiores ao mínimo de $60 \% .{ }^{20}$ Ao final das análises estatísticas, o nível de significância utilizado foram os fatores com autovalores maiores que 1,0.

Tabela 4 - Descrição de fator: autovalores e percentual explicado.

\begin{tabular}{cccc}
\hline Fator & Autovalor & $\begin{array}{c}\text { Percentual da } \\
\text { variância }\end{array}$ & $\begin{array}{c}\text { Percentual da variância } \\
\text { acumulada }\end{array}$ \\
\hline $\mathbf{1}$ & $\mathbf{7 , 2 9 3}$ & 42,899 & 42,899 \\
$\mathbf{3}$ & 1,595 & 9,380 & 52,279 \\
$\mathbf{4}$ & 1,202 & 7,073 & 59,352 \\
$\mathbf{5}$ & 1,081 & 6,357 & 65,709 \\
$\mathbf{6}$ & 0,916 & 5,388 & 71,098 \\
$\mathbf{7}$ & 0,813 & 4,782 & 75,879 \\
$\mathbf{8}$ & 0,705 & 4,148 & 80,027 \\
$\mathbf{9}$ & 0,596 & 3,508 & 83,535 \\
$\mathbf{1 0}$ & 0,549 & 3,230 & 86,765 \\
$\mathbf{1 1}$ & 0,530 & 3,119 & 89,884 \\
$\mathbf{1 2}$ & 0,413 & 2,429 & 92,313 \\
$\mathbf{1 3}$ & 0,352 & 2,071 & 94,384 \\
$\mathbf{1 4}$ & 0,300 & 1,766 & 96,150 \\
$\mathbf{1 5}$ & 0,278 & 1,634 & 97,784 \\
$\mathbf{1 6}$ & 0,176 & 1,034 & 98,817 \\
$\mathbf{1 7}$ & 0,122 & 0,716 & 99,533 \\
\hline & 0,079 & 0,467 & 100,000 \\
\hline & & & \\
\hline & & & \\
\hline
\end{tabular}


Tabela 5 - Distribuição de frequência dos valores do OHIP-14 por item aplicado.

\begin{tabular}{lcccccc}
\hline Pergunta: ... devido a & Nunca & Quase & Ocasionalmente & Pouco & Muito & Mé \\
problemas com seus & $\mathbf{( 0 )}$ & nunca & (2) & frequente & frequente & dia \\
dentes, boca ou prótese? & & (1) & & (3) & (4) &
\end{tabular}

\begin{tabular}{lcccccr}
\hline & $\mathbf{N}(\%)$ & $\mathbf{N}(\%)$ & $\mathbf{N}(\%)$ & $\mathbf{N}(\%)$ & $\mathbf{N}(\%)$ & \\
\hline 1 - Você tem dificuldade & $35(70)$ & $6(12)$ & $5(10)$ & $2(4)$ & $2(4)$ & 0,60
\end{tabular}

para pronunciar alguma

palavra...

\begin{tabular}{|c|c|c|c|c|c|c|}
\hline $\begin{array}{l}2 \text { - Você sente que seu } \\
\text { paladar piorou... }\end{array}$ & $24(48)$ & $3(6)$ & $9(20)$ & $4(10)$ & $10(16)$ & 1,40 \\
\hline $\begin{array}{l}3 \text { - Você tem sofrido } \\
\text { dores na sua boca ou } \\
\text { dentes... }\end{array}$ & $13(26)$ & $4(10)$ & $16(32)$ & $3(8)$ & $14(24)$ & 1,94 \\
\hline $\begin{array}{l}4 \text { - Você sente } \\
\text { dificuldade para comer } \\
\text { algum alimento... }\end{array}$ & $17(34)$ & $4(8)$ & $7(14)$ & $5(10)$ & $17(34)$ & 2,02 \\
\hline $\begin{array}{l}5 \text { - Você se sente } \\
\text { inibido... }\end{array}$ & $18(36)$ & $2(4)$ & $2(4)$ & $8(16)$ & $20(40)$ & 2,20 \\
\hline $\begin{array}{l}6 \text { - Você tem se sentido } \\
\text { tenso... }\end{array}$ & $12(24)$ & $3(6)$ & $5(10)$ & $2(4)$ & $28(56)$ & 2,62 \\
\hline $\begin{array}{l}7 \text { - Sua dieta tem sido } \\
\text { insatisfatória... }\end{array}$ & $24(48)$ & $8(4)$ & $11(22)$ & $3(6)$ & $8(16)$ & 1,34 \\
\hline $\begin{array}{l}8 \text { - Você tem } \\
\text { interrompido suas } \\
\text { refeições... }\end{array}$ & $21(42)$ & $12(24)$ & $7(14)$ & $7(14)$ & $3(6)$ & 1,18 \\
\hline $\begin{array}{l}9 \text { - Você sente } \\
\text { dificuldade em relaxar... }\end{array}$ & $\begin{array}{c}19 \\
(38)\end{array}$ & $8(16)$ & $6(12)$ & $6(12)$ & $11(22)$ & 1,64 \\
\hline $\begin{array}{l}10 \text { - Você tem se sentido } \\
\text { embaraçado... }\end{array}$ & $18(36)$ & $3(6)$ & $5(10)$ & $7(14)$ & $17(34)$ & 2,04 \\
\hline $\begin{array}{l}11 \text { - Você tem se sentido } \\
\text { irritado com outras } \\
\text { pessoas... }\end{array}$ & $23(48)$ & $3(6)$ & $6(12)$ & $8(16)$ & $10(18)$ & 1,50 \\
\hline $\begin{array}{l}12 \text { - Você tem tido } \\
\text { dificuldades de realizar } \\
\text { seus trabalhos diários... }\end{array}$ & $25(50)$ & $2(4)$ & $5(12)$ & $8(16)$ & $10(18)$ & 1,48 \\
\hline $\begin{array}{l}13 \text { - Você tem sentido a } \\
\text { vida menos satisfatória... }\end{array}$ & $\begin{array}{c}22 \\
(44)\end{array}$ & $1(2)$ & $6(12)$ & 2(4) & $19(38)$ & 1,90 \\
\hline
\end{tabular}


O escore total do OHIP-14 foi de 22,6, o que corresponde a 40,36\% do valor máximo (Tabela 5).

Tabela 6 - Distribuição do impacto do OHIP-14 categorizado por dimensão e total aplicado em ex-moradores de rua acolhidos. Uberlândia, 2014.

\begin{tabular}{lcccc}
\hline Dimensão & Sem impacto & Com impacto \\
\hline & $\mathbf{N}$ & $\%$ & $\mathbf{N}$ & $\%$ \\
\hline Limitação funcional & 36 & 72 & 14 & 28 \\
\hline Dor física & 23 & 46 & 27 & 54 \\
Desconforto psicológico & 15 & 30 & 35 & 70 \\
Incapacidade física & 32 & 64 & 18 & 36 \\
Incapacidade psicológica & 23 & 46 & 27 & 54 \\
Incapacidade social & 23 & 46 & 27 & 54 \\
Deficiência & 28 & 56 & 22 & 44 \\
OHIP total & 11 & 22 & 39 & 78 \\
\hline
\end{tabular}

Observou-se que $78 \%$ dos indivíduos perceberam impacto das condições bucais na sua qualidade de vida em pelo menos uma das sete dimensões descritas a seguir: limitação funcional, dor física, desconforto psicológico, incapacidade física, incapacidade psicológica, incapacidade social e deficiência. A dimensão que apresentou maior prevalência de impacto (70\%) foi desconforto psicológico.

\section{Dıscussão}

Por meio dos resultados obtidos por este trabalho, foi possível conhecer os aspectos envolvidos na autopercepção da saúde bucal, relacionada à qualidade de vida, que mais predominaram na população de moradores de rua estudados. As dimensões mais afetadas foram desconforto psicológico (70\% sentiramse inibidos e tensos), incapacidade psicológica (54\% sentiram-se com dificuldade para relaxar e embaraçado), incapacidade social (54\% sentiram irritação e dificuldade de realizar trabalhos diários) e dor física (54\% sentiram dores na boca ou dentes e dificuldade para comer). $\mathrm{Na}$ Tabela 6 , observa-se grande impacto das condições bucais na qualidade de vida dos entrevistados.

Esta pesquisa corrobora com o estudo de Silva et al., ${ }^{21}$ que apontaram que 0 desconforto psicológico, dor física e inabilidade psicológica foram as dimensões de maior impacto na qualidade de vida, semelhante aos dados encontrados em outros estudos, em que se avaliou o impacto da saúde bucal na qualidade de vida. ${ }^{22}$

Em estudo realizado na Suécia, no qual se avaliou por meio do OHIP o impacto na qualidade de vida de moradores de rua e de ex-moradores acolhidos em abrigos, foi demonstrado que $70 \%$ deles sentiram-se inibidos e tensos, semelhante ao presente estudo feito em 2010. ${ }^{23}$

A maioria dos indivíduos entrevistados escolheu a opção de resposta "muito frequente" para a questão relacionada à experiência de se sentir tenso por causa de problemas com os dentes, boca ou prótese, relacionada à dimensão desconforto psicológico ${ }^{15}$. De Palma e Nordenram (2005) relataram que uma aparência bucal restabelecida aumenta a sensação de competência social, função social, autoconfiança e autoestima. ${ }^{24}$ 
No que diz respeito à incapacidade social, os ex-moradores de rua se mostraram com uma fragilidade de vínculos familiares, sociais, e nas relações interpessoais em geral. Entretanto, há relato na literatura de que os indivíduos que moram na rua, mesmo tendo níveis semelhantes de doenças bucais, embora não tratada, experimentam elevado impacto de sua condição dental, em comparação com a população de alojados. Um estudo qualitativo em Londres, realizado em 2007, sugere que a aparência oral e geral tem um papel de grande importância na vida das pessoas que vivem sem abrigo. Os participantes relataram que uma aparência bucal e geral bem conservada, protegeu-os do estigma social e dos sentimentos de vergonha e embaraço. ${ }^{25}$

Como não há relatos na literatura da avaliação do impacto da saúde bucal na qualidade de vida em ex-moradores de rua, buscaram-se trabalhos realizados com moradores de rua, os quais avaliaram a qualidade de vida também utilizando o OHIP. Em estudo do Reino Unido 1993, 91\% dos moradores de rua relataram pelo menos um impacto e $52 \%$ descreveram cinco itens ou mais. Apenas $11 \%$ relataram nenhum impacto. ${ }^{26}$

Em um estudo realizado por Conte et al. (2006), com adultos desabrigados em Newark, verificou-se que $86 \%$ das pessoas relataram impactos de saúde bucal negativos. ${ }^{27}$ Estudos de pessoas desabrigadas na Irlanda do Norte, em 2007, sugeriram que a saúde bucal foi uma fonte de diminuição da qualidade de vida. ${ }^{28}$

A rotina foi significativamente afetada pela condição de saúde bucal, deixando-os irritados e com dificuldades para realizar os trabalhos diários, o que corrobora com os achados de outros estudos. ${ }^{9}$

Em relação à dimensão dor física, mais da metade dos ex-moradores de rua tem dores na boca e dificuldade para comer. Em comparação, uma pesquisa no Reino Unido, realizada em 1993, indicou que as pessoas "sem-teto" têm pior saúde mental e física e morrem mais cedo que os seus homólogos alojados. Relativamente suas condições são agravadas por problemas de acesso a cuidados de saúde e meios de atendimento inadequados. ${ }^{26}$

Quase metade dos ex-moradores de rua sente que a vida é menos satisfatória, e eles se sentem totalmente incapazes de suas obrigações. Os fatores de vulnerabilidade psicossocial levam o indivíduo a ter completa desesperança em relação à sua própria vida e ao seu futuro. ${ }^{24}$

No que se refere à alimentação, $40 \%$ dos entrevistados já interromperam as refeições e tiveram dieta insatisfatória. Sendo assim, problemas com a boca, dentes ou prótese podem ser referenciados como fortes preditores de impacto que, além de causar danos funcionais $(32 \%$ tem dificuldade de pronunciar palavras e sentem mudança no paladar), são capazes de desequilibrar a organização psíquica e social dos indivíduos. A baixa prevalência de indivíduos relatando problemas com a fala, pode ser devido a sentimentos de solidão e abandono, por não terem ninguém para conversar. ${ }^{29}$

É interessante observar que a resposta mais frequente para todas as perguntas do OHIP-14 foi nunca, o que corrobora com os resultados obtidos em trabalhos que apontaram para o fato de as pessoas terem precária percepção de seus problemas bucais, como se estes fossem inevitáveis. É amplamente inferido na literatura que moradores de rua são apáticos com relação à sua saúde oral e ou a consideram como prioridade baixa. ${ }^{30}$

A avaliação da qualidade de vida dos ex-moradores de rua da Casa de Acolhimento Fraterno Santa Gemma Galgani mostrou que os domínios com piores escores foram desconforto psicológico, incapacidade psicológica, incapacidade social e dor física. A ausência de 
um companheiro(a), a falta de participação ativa na comunidade, a falta de responsabilidades sociais, a falta de recursos disponíveis para enfrentar adversidades, a falta de apoio social, a desesperança em relação à vida, as condições de alimentação, a autodesvalorização e a falta de dentes são fatores existentes na vida do grupo de ex-moradores de rua que podem contribuir para a pior percepção da saúde bucal na qualidade de vida.

\section{Conclusão}

A amostra desta pesquisa, ex-moradores de rua de Uberlândia-MG, foi subsídio para a revisão e o fortalecimento de políticas públicas destinadas aos moradores de rua, à medida que contribuem para reflexões acerca da necessidade de garantir a esses indivíduos direitos que configurem o exercício da cidadania. O índice OHIP-14 mostrou a existência de impacto da saúde bucal na qualidade de vida dos ex-moradores de rua, tendo sido maior nas dimensões desconforto psicológico, dor física, incapacidade psicológica e incapacidade social.

\section{REFERÊNCIAS}

1 Varanda W., Adorno RCF. Descartáveis urbanos: discutindo a complexidade da população de rua e o desafio para políticas de saúde. Saúde e Sociedade, 2004, jan., 13(1): 56-69.

2 Rosa AS, Secco MG, Brêtas ACP. O cuidado em situação de rua: revendo o significado do processo saúde-doença. Rev Bras Enferm, 2006, maio, 59(3): 331-336.

3 Bordignon JS, Silveira CCS, Delvivo EM, Araújo EM, Lasta LD, Weiller TH. Adultos em situação de rua: Acesso aos Serviços de Saúde e Constante Busca Pela Ressocialização. Revista Contexto \& Saúde, 2011, jan., 10(20): 629634.

4 Vasconcellos EM. Educação Popular e a Atenção à Saúde da Família. Editora Hucitec, 1999.
5 Romanholi RMZ, Cyrino EG. A visita domiciliar na formação de médicos: da concepção ao desafio do fazer. Interface (Botucatu), 2012, 16(42): 693-705.

6 Araújo ME. Palavras e silêncios na educação superior em odontologia. Ciência \& Saúde Coletiva, 2006, 11(1): 179-182.

7 Sampaio TMV. O movimento da vida e seus desafios à Extensão Universitária. Revista Dialogos: Construção Conceitual de Extensão e Outras Reflexões Significativas, 2010, 14(1):25-32.

8 Filho NA. O conceito de saúde: ponto cego da epidemiologia? R. bras. Epidemiol., 2000, $3(1): 4-20$.

9 Slade D., Spencer AJ. Development and evaluation of the Oral Health Impact Profile. Community Dent Health, 1994, 11(1):3-11.

10 Walter MH, Woronuk JI, Tan HK, Lenz U., Koch $\mathrm{R}$., Boening KW. Oral health related quality of life and its association with sociodemographic and clinical findings in 3 northern outreach clinics. J Can Dent Assoc., 2007, 73(2):153.

11 Katz JN, Larson MG, Phillips CB, Fossel AH, Liang $\mathrm{MH}$. Comparative measurement sensitivity of short and longer health status instruments. Med. Care., 1992, 30:917-925.

12 Maryane OC, João FRN. Qualidade de vida: um instrumento para promoção de saúde. Revista Baiana de Saúde Pública, 2008, mai./ago., 32(2): 232-240.

13 Lundegren N., Axtelius B., Hakansson J., Akerman S. Dental treatment need among 20 to 25-year-old Swedes: discrepancy between subjective and objective need. Acta Odontol Scand., 2004, 62(2):91-96.

14 Vargas AMD, Paixão HH. Perda dentária e seu significado na qualidade de vida de adultos usuários de serviço público de saúde bucal do Centro de Saúde Boa Vista, em Belo Horizonte. Cienc Saude Coletiva, 2005, 10(4):1.0151.024.

15 Petersen PE, Bourgeois D., Ogawa H., Estupinan-Day S., Ndiaye C. The global burden of oral diseases and risks to oral health. Bull World Health Organ., 2005; 83:661-9.

16 Slade GD. Derivation and validation of a shortform oral health impact profile. Community Dent Oral Epidemiol. 1997, 25(4): 284-290. 
17 Leão AT, Locker D., Antunes JLF, Peres MA. Fundamentos de odontologia. Epidemiologia da saúde bucal, Guanabara Koogan, 2006, 260-268.

18 Bianco VC, Lopes ES, Borgato MH, Silva PM, Marta SN. O impacto das condições bucais na qualidade de vida de pessoas com cinquenta ou mais anos de vida. Ciência Saúde Coletiva, 2010, 15(4):2.165-2.172.

19 Oliveira BH, Nadanovsky P. Psychometric properties of the Brazilian version of the oral health impact profile-short form. Community Dentistry and Oral Epidemiology, 2005, 33: 307-314.

20 Hair J., Black WC, Babin BJ, Anderson RE, Tatham RL. Multivariate Data Analysis. 6nd ed. Upper Saddle River (NJ): Pearson Prentice Hall, 2006.

21 Silva MES, Villaça EL, Magalhães CS, Ferreira EF. Impacto da perda dentária na qualidade de vida. Ciênc Saúde Coletiva, 2010, 15:841850.

22 Locker D., Quiñonez C. To what extent do oral disorders compromise the quality of life? Community Dent Oral Epidemiol. 2011, 39(1):3-11.

23 Daly B., Newton T., Batchelor P., Jones K. Oral health care needs and oral health-related quality of life (OHIP-14) in homeless people. Community Dent Oral Epidemiol. 2010. _ 2010 John Wiley \& Sons A S.
24 De Palma P., Northareren P. The perceptions of homeless people in Stockholm concerning oral health and consequences of oral treatment: a qualitative study. Spec Care Dent., 2005, 25:289-95.

25 Daly B. The Oral Health Needs of Homeless People. PhD Thesis, London: University of London, 2007.

26 Balazs J. Health care for single homeless people. In: Fisher K., Collins J., editors. Homelessness, Health Care and Welfare Provision. London: Routledge, 1993, 51-93.

27 Conte M., Broder HL, Jenkins G., Reed R., Janal MN. Oral health, related behaviours and oral health impacts among homeless adults. $\mathbf{J}$ Public Health Dent., 2006, 66:276-8.

28 Collins J., Freeman R. Homeless in North and West Belfast: an oral health needs assessment. Brit Dent J., 2007, 202:1-6.

29 Brenes-Camacho G. Favourable changes in economic well-being and self-rated health among the elderly. Social Science \& Medicinev., 2011, 72(8):1.228-1.235.

30 Jago JD, Sternberg G., Westerman B. Oral health status of homeless men in Brisbane.

Aust Dent., 1994, 29:184-8.

Submetido em: 14-5-2016

Aceito em: 17-10-2016 